\title{
Deformation Analysis of Reinforced Concrete using Neutron Imaging Technique
}

\author{
T. Koyama ${ }^{1}$, K. Ueno ${ }^{2}$, M. Sekine ${ }^{1}$, Y. Matsumoto ${ }^{3, a}$, T. Kai ${ }^{4, b}$, T. Shinohara ${ }^{4, c}, H$. \\ likura $^{5, \mathrm{~d}}, \mathrm{H}$. Suzuki ${ }^{5, \mathrm{e}^{*}}$ and M. Kanematsu ${ }^{2, \mathrm{f}}$
}

${ }^{1}$ Graduate School of Science and Technology, Tokyo University of Science, Noda, Chiba, Japan

${ }^{2}$ Faculty of Science and Technology, Tokyo University of Science, Noda, Chiba, Japan

${ }^{3}$ Research Center for Neutron Science and Technology, Comprehensive Research Organization for Science and Society, Tokai, Ibaraki, Japan

${ }^{4} J$-PARC Center, Japan Atomic Energy Agency, Tokai, Ibaraki, Japan

${ }^{5}$ Materials Sciences Research Center, Japan Atomic Energy Agency, Tokai, Ibaraki, Japan

ay_matsumoto@cross.or.jp, btetsuya.kai@j-parc.jp, ${ }^{c}$ takenao.shinohara@j-parc.jp,

diikura.hiroshi@jaea.go.jp, ${ }^{\mathrm{e}}$ suzuki.hiroshi07@jaea.go.jp, ${ }^{f}$ manabu@rs.noda.tus.ac.jp

Keywords: Neutron Imaging, Reinforced Concrete, Deformation Analysis, Image Analysis

\begin{abstract}
We suggest a novel method to observe internal deformation of concrete using a neutron transmission imaging technique. In order to visualize the internal deformation of concrete, cement paste markers containing $\mathrm{Gd}_{2} \mathrm{O}_{3}$ powder were dispersed two-dimensionally around the ferritic deformed rebar in reinforced concrete. Displacement of the neutron transmission image of the $\mathrm{Gd}$ marker was evaluated by a change in the position of the marker as a function of the travel distance of the vertical sample stage, and it was successfully evaluated to within approximately $\pm 0.1 \mathrm{~mm}$ accuracy by analyzing selected markers with higher contrast and circularity. Furthermore, concrete deformation under pullout loading to the embedded rebar was evaluated in the same way and compressive deformation of concrete was successfully observed by analyzing the displacement of the markers. The results obtained in this study bring beneficial knowledge that the measurement accuracy of the marker displacement can be improved by choosing spherical-shaped markers and by increasing the contrast of markers.
\end{abstract}

\section{Introduction}

Reinforced concrete (RC), widely utilized for various architectural and civil engineering structures, is well known as a composite structure in which concrete with relatively low tensile strength and ductility is strengthened by reinforcements such as steel rods (rebars) with high tensile strength and ductility. The structural performance of $\mathrm{RC}$ is generally derived from the bond resistance between rebar and concrete. It has been demonstrated in previous studies that the neutron diffraction technique can be an alternative method to conventional strain gauges for evaluation of bond resistance by measuring the stress distribution of rebar embedded in concrete [1-3].

On the other hand, it is also important to evaluate the deformation behavior of concrete around the embedded rebar in order to discuss the mechanism of bond degradation between concrete and rebar for the RC structure. However, it is difficult to apply the neutron diffraction technique to the measurement of strain in concrete since high background noise scattering from hydrogen within the concrete makes diffraction measurements in concrete difficult. Alternatively, image analysis techniques such as a lattice method [4] and digital image correlation (DIC) [5], are commonly utilized for evaluating deformation of concrete quantitatively. They can assess concrete surface 
deformation by analyzing image contrast or marker displacement taken by a high resolution camera. In this study, therefore, we aim to develop a novel method to observe internal deformation of concrete using the neutron transmission imaging technique combined with a lattice method.

\section{Experimental Procedure}

Reinforced Concrete Specimen. Fig. 1 shows the schematic illustration of the RC specimen used in this study. A ferritic steel deformed-bar with a nominal $12.7 \mathrm{~mm}$ diameter to JIS3112 standard was embedded in a rectangular concrete specimen of size $50 \times 50 \times 130 \mathrm{~mm}^{3}$. The embedded length of rebar was $100 \mathrm{~mm}$, and unbonded region of $30 \mathrm{~mm}$ in length was artificially introduced by surrounding the rebar with a polyvinyl chloride (PVC) pipe. In order to visualize the internal deformation of concrete around the embedded rebar, the cement paste markers containing $34 \mathrm{wt} . \%$ $\mathrm{Gd}_{2} \mathrm{O}_{3}$ powder (hereafter called "Gd marker") were dispersed two-dimensionally covering $15 \%$ of the area of the A-A' cross section. The Gd markers were obtained by pulverizing a paste cylinder of $\phi 50 \times 100 \mathrm{~mm}^{3}$ having a specific $\mathrm{Gd}_{2} \mathrm{O}_{3}$ content and classifying the particle size into 0.6 to 1.7 $\mathrm{mm}$. The RC specimen was demolded 72 hours after placing and then cured in water for 7 days. In order to reduce water in concrete that causes neutron attenuation by hydrogen, the RC specimen was placed in a constant temperature $\left(20 \pm 1{ }^{\circ} \mathrm{C}\right)$ and humidity $(60 \pm 5 \mathrm{RH} \%)$ room for 24 hours, and then was dried at $60{ }^{\circ} \mathrm{C}$ for 4 days before the neutron experiment.

Neutron Optical System. Fig. 2 shows the optical layout utilized in this study. The RC specimen mounted on the loading device, composed of a hydraulic jack and a load cell, was set up on a sample stage of BL22, RADEN [6], in the Materials and Life Science Experimental Facility (MLF) of the Japan Proton Accelerator Research Complex (J-PARC). RADEN is a next generation pulsed-neutron instrument, energyresolved neutron imaging facility. The beam power of J-PARC MLF for this experiment was $150 \mathrm{~kW}$, and the neutron flux at the sample position was estimated to be 5.0 $\times 10^{5} \mathrm{n} / \mathrm{cm}^{2} / \mathrm{sec}$. The L/D ratio was set to 1000 . The incident neutrons scattered and absorbed by the RC specimen were taken by a cooled CCD camera after being converted to visible light by a scintillator. The distance between the scintillator and the sample edge was set to 200 $\mathrm{mm}$ so as to reduce the influence of scattering from the specimen. A total of four images each with a 15 minute exposure time were taken with the same measurement configuration. The resolution of the transmission image was $2048 \times 2048$ pixels (16 bit), and the spatial resolution was approximately equivalent to be $0.2 \mathrm{~mm}$. A displacement transducer with an accuracy of $0.01 \mathrm{~mm}$ was set up on the RC specimen to measure the displacement of the edge of the embedded rebar under pullout loading.

Experimental Condition. The analytical condition was, at first optimized by evaluating the displacement of the Gd marker images as a function of the travel distance of the sample stage in order to determine the position of the $\mathrm{Gd}$ marker on the transmission image accurately. The position of the Gd marker in a neutron transmission image of the RC specimen was evaluated by an image analysis technique using an open source image processing program,

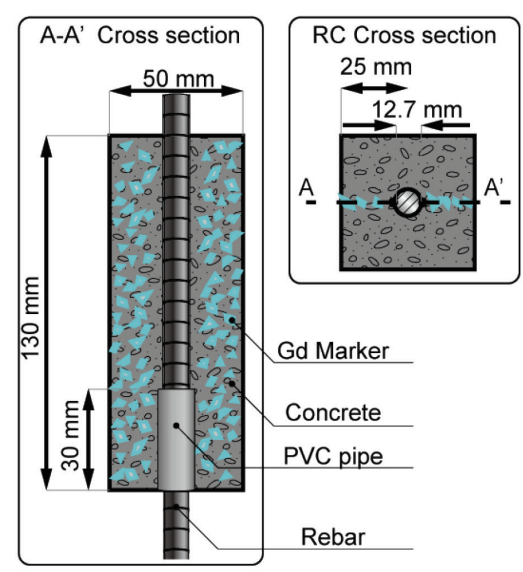

Fig. 1 Schematic illustration of the $R C$ specimen used in this study.

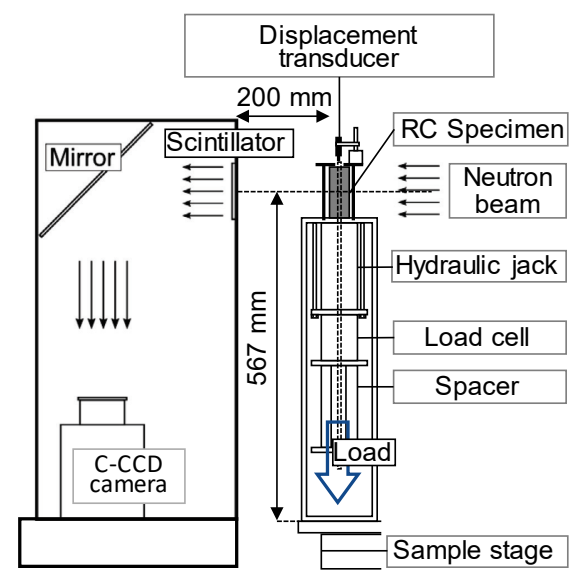

Fig. 2 Schematic opticul layoui for imaging experiment. 
ImageJ $1.51 \mathrm{n}[7]$. The transmission images were taken at $0.0,0.1,0.2,0.3,0.31,0.33,0.36$ and 0.4 $\mathrm{mm}$ from the given original position by translating the sample vertically.

Secondly, the deformation of the RC specimen was measured under compressive deformation by analyzing the displacement of the Gd marker on the transmission image in order to verify the optimum condition of the image analysis determined by the above displacement measurement. The pullout loadings at 5, 10, 15, 20, 25 and $30 \mathrm{kN}$ were applied to the rebar as shown in Fig. 2, making compressive deformation in the concrete part of the specimen. Pull out loading was manually controlled by the measured value of load cell installed in the loading device. The load on the rebar decreased drastically just after stopping the loading due to creep deformation. However, the displacement of the rebar measured by a displacement transducer was negligibly small, which means that concrete deformation around rebar was quite small during creep. Therefore, the transmission images started to be taken after waiting for 5 to 10 minutes after applying the load.

\section{Results and Discussion}

Transmission Image. Figure 3a shows a neutron transmission image of the RC specimen taken by RADEN. The Gd markers dispersed in the concrete matrix can be recognized in the image clearly. The dark area at the bottom of the center of the image represents the PVC pipe covering the rebar, as shown in Fig. 3a. In addition, the image contrast between concrete matrix and the embedded rebar can be observed as well.

Analysis of Gd Marker Position. The flow of the image analysis is shown in Fig. 3. The image analysis by Image J was conducted in an area of $940 \times 1956$ pixels as shown in Fig. 3a. At first, the transmission image was normalized by the incident neutron flux and the shade image (Fig. 3b). And then, the median filter was applied to some transmission images taken at the same sample position or at the same loading, in order to remove white spot noise and to smooth the image. After applied the median filter, a Fourier transform was applied and low frequencies in the transmission image were filtered by masking obtained by a $2 \mathrm{D}$ Fourier power spectrum less than 15 pixels in radius (Fig. 3c). After that, the spectrum obtained by excluding the low-frequencies was converted to the transmission image using an inverse Fourier transform procedure. This is known as a general high-pass filter, which can highlight the Gd marker images by reducing the background contrast variation representing the rebar and the PVC pipe. The transmission image obtained by the high-pass filter was binarized by defining an appropriate threshold (Fig. 3d). And then, using a morphological process, the spot noise on the obtained binary image was removed and the Gd marker images were shaped. Eventually, the Gd marker images were successfully extracted from the original transmission image as shown in Fig.
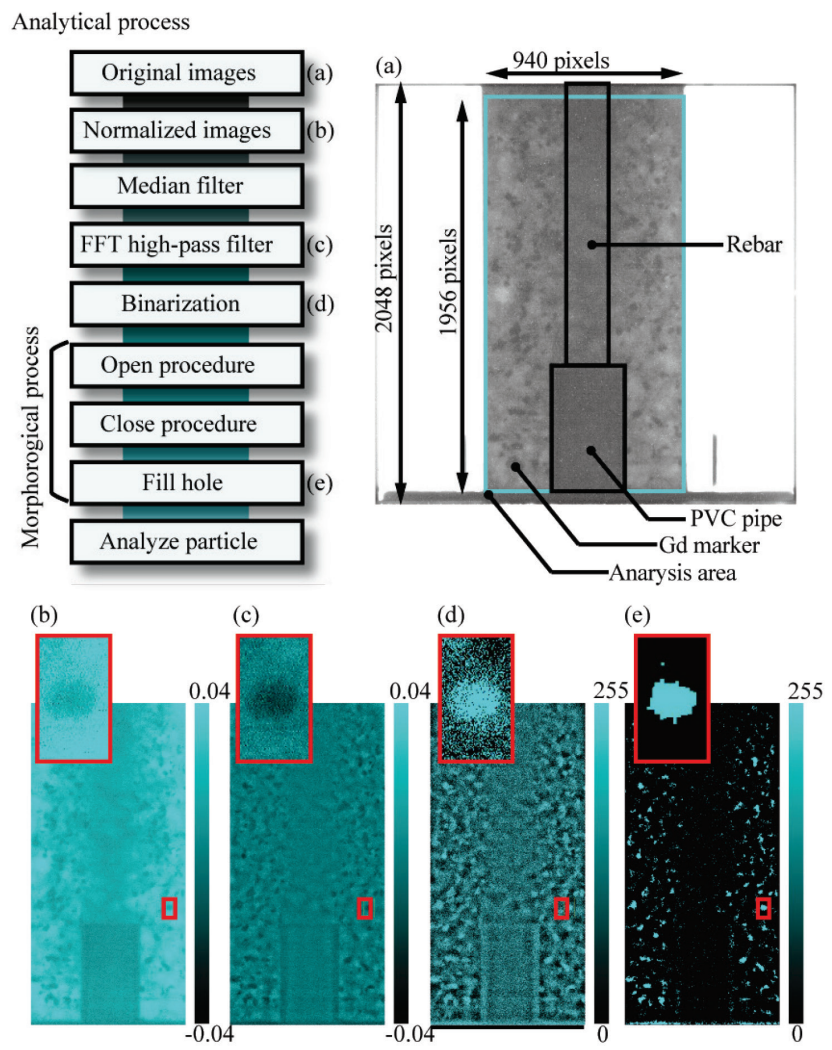

Fig. 3 Transmission image of the RC specimen (a), and the images for each process, (b), (c), (d) and (e), in the analytical procedure.

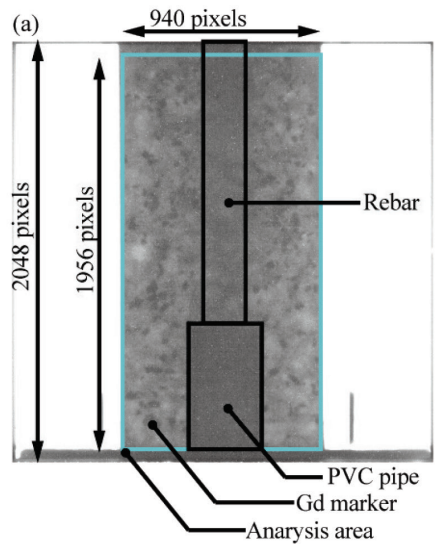

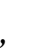


the center of mass (COM) for each marker was determined by analyzing particles using ImageJ. In the analysis, particles less than 300 pixel $^{2}$ in size (equivalent to the area of a circle with a diameter of $1 \mathrm{~mm}$ ) were omitted to distinguish from the noise. Furthermore, the particles obviously different from the Gd markers were also regarded as noise and were manually omitted as well.

Evaluation of Marker Displacement. Displacement of the Gd marker image was evaluated by a change in the COM of the marker as a function of the travel distance of the vertical sample stage. As a result, the variation of the Gd marker displacement is quite scattered as shown in Fig. 4. The average displacement in Fig. 4b shows a linear variation in proportion to travel distance of the sample stage, with a standard deviation of \pm 4.5 pixels (equivalent to $\pm 0.23 \mathrm{~mm}$ ). This error might be due to the unstable shape of the $\mathrm{Gd}$ marker image. For instance, there is a case that the area, $A_{0}$, of the Gd marker image taken at an initial position of the sample stage is different from the area, $A_{i}$, of that taken at the next position of the sample stage (insertion individual marker images in Fig. 5a). This would be due to insufficient statistics compared
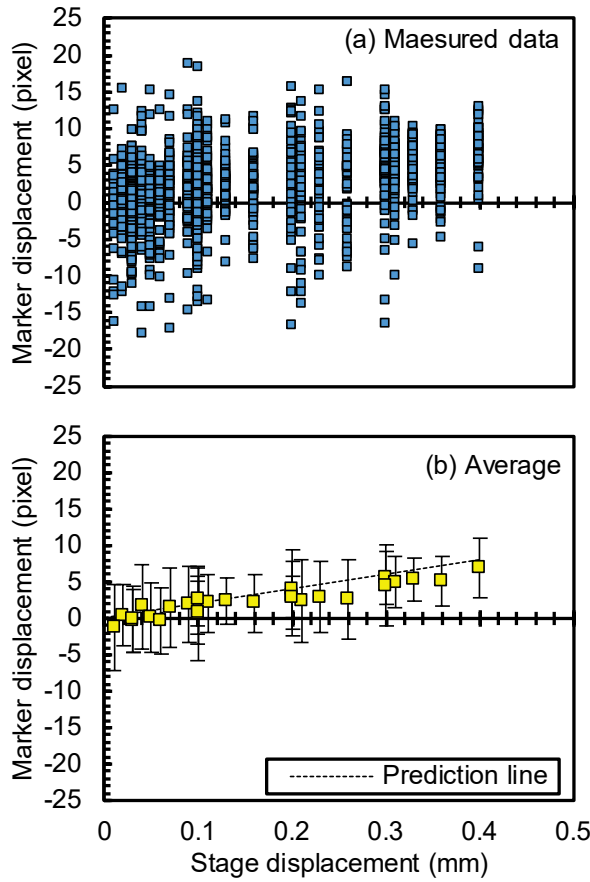

Fig. 4 The relation between marker and stage displacements. Prediction line in (b) corresponds to movement of the stage. with the contrast of the Gd marker image. The relation between the amounts of scattering and a threshold that gives an extraction limit of the Gd marker images were evaluated as a low-threshold to be defined by an area ratio, i.e. $A_{i} / A_{0}\left(A_{0}>A_{i}\right)$ or $A_{0} / A_{i}$ $\left(A_{i}>A_{0}\right)$. If choosing only Gd marker images that exceed a threshold, an increase in the area ratio, that is $A_{i}$ approaches $A_{0}$, tends to decrease the scattering although the number of selectable Gd marker images decreases, as shown in Fig. 5a. Here, the standard deviation in Fig. 5a exhibits an average value of error bars taken at all sample displacements, and the number of the Gd marker images were counted in the transmission image taken at the initial stage position. Therefore, it was concluded that a change in the area of the Gd marker image, induced by an insufficient contrast, influences the accuracy of evaluation of the Gd marker displacement.
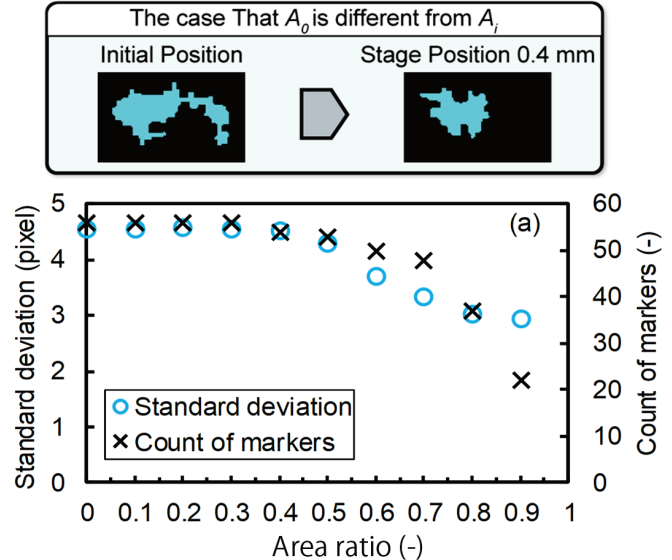

The case that the Gd marker is complex shape
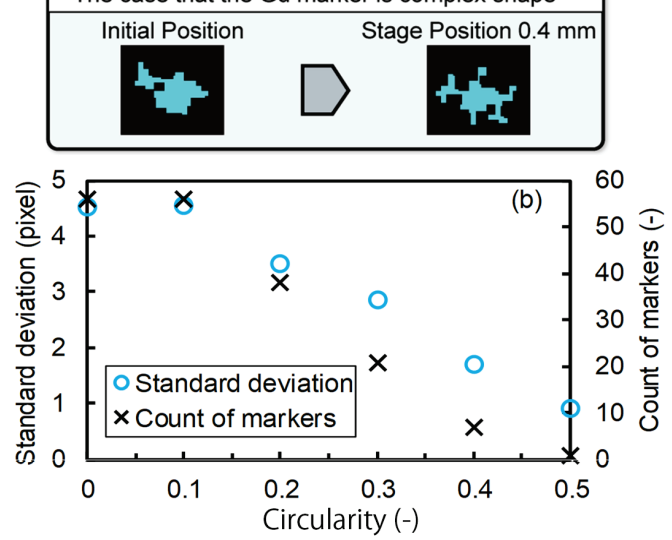

Fig. 5 The relations (a) between standard deviation and area ratio and (b) between standard deviation and circulality. Insertion images show examples of the change in the marker shape. 
On the other hand, there is a case that Gd marker images with complex shape changes to a different shape at the next position of the sample stage (insertion individual marker images in Fig. 5b). Here, the relation between the standard deviation and the $\mathrm{Gd}$ marker images circularity, which were computed by ImageJ [7], was investigated. If choosing only Gd marker images above a given circularity, an increase in the circularity omits markers with a complex shape thereby decreasing the standard deviation as shown in Fig. 5b. Therefore, it was concluded that a change in the shape of the complex Gd marker image, induced by insufficient contrast, influences the accuracy of determination of the Gd marker displacement. Accordingly, the measurement accuracy of the displacement of the $\mathrm{Gd}$ marker might be improved by choosing the Gd marker images close to a circler shape.

Fig. 6 shows the Gd marker displacement evaluated at a low-threshold defined as an area ratio of 0.8 and a circularity of 0.3 . At this threshold, the number of $\mathrm{Gd}$ markers decreased from 56 to 21 . It can be found by comparing this result with Fig. 5 that the plots with large error were preferentially omitted by defining an appropriate threshold of area ratio and circularity. This result shows the scattering of \pm 2.2 pixels (equivalent to $\pm 0.11 \mathrm{~mm}$ ), which is approximately half of the result in Fig. 4. Therefore, the measurement accuracy of the Gd marker displacement can be improved by choosing a spherical shaped Gd marker, and by increasing the contrast of the Gd marker by increasing the amount of $\mathrm{Gd}_{2} \mathrm{O}_{3}$ powder contained in the marker and also by increasing statistical accuracy.

Deformation Analysis. Concrete deformation of the RC specimen was measured under pullout loading. The concrete part along the bonded region integrally shifts vertically toward the loading direction followed by the compressive deformation along the unbounded region. The transmission image was evaluated at a low-threshold defined as an area ratio of 0.8 and a circularity of 0.3 . Applying this threshold, the number of acceptable markers at $5,10,15,20,25$ and $30 \mathrm{kN}$ is $3,8,22,12,0$ and 6 , respectively. As shown in Fig. 7, the Gd marker images move toward the loading direction proportionally to applied loading. Furthermore, the displacement of the Gd marker images are always evaluated to be slightly smaller than the displacement of rebar measured by a displacement transducer, while their trends agree well within the error bars. This small difference might be caused by asymmetric distortion of the RC specimen under pullout loading. Selecting the appropriate Gd marker with higher contrast and circularity enables us to analyze deformation of concrete accurately.
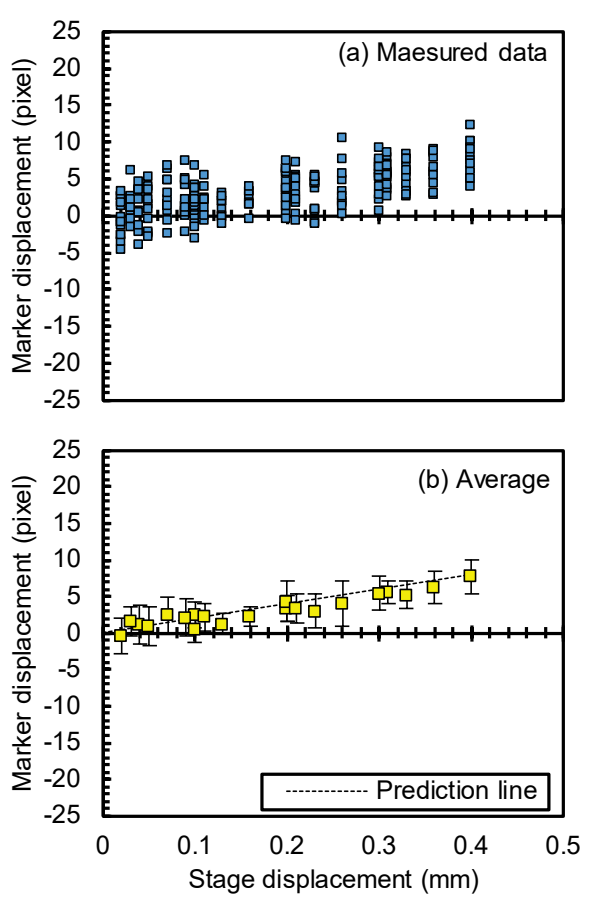

Fig. 6 The relation between marker and stage displacements obtained by defining threshold as an area ratio of 0.8 and a circularity of 0.3 .
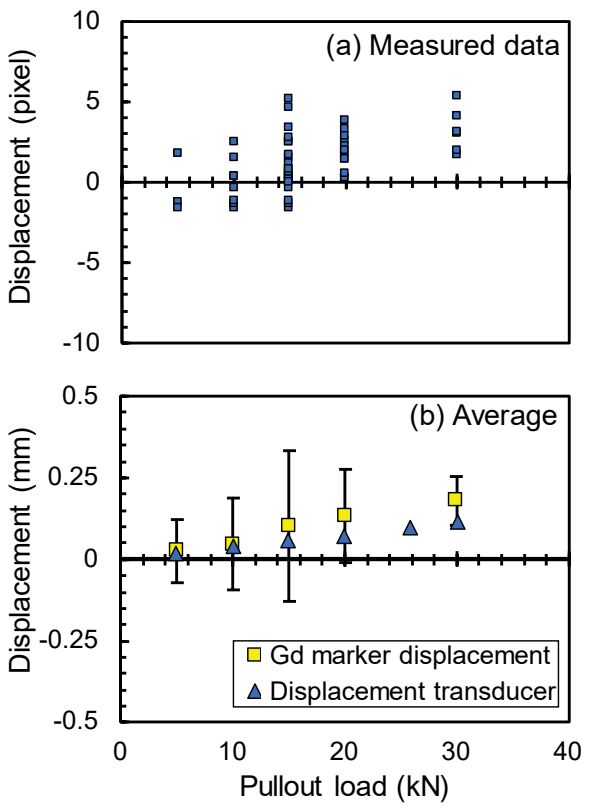

Fig. 7 The relation between marker displacement and applied loading, compared with the displacement measured by a displacement tranceducer. 


\section{Summary}

We have suggested a novel method to observe internal deformation of concrete by the neutron transmission imaging technique with image analysis. In order to visualize the internal deformation of concrete around the embedded rebar, the displacement of the cement paste markers containing 34 wt. $\% \mathrm{Gd}_{2} \mathrm{O}_{3}$ powder, dispersed two-dimensionally in the reinforced concrete samples were evaluated. Displacement of the Gd marker image was evaluated by a change in the position of the marker as a function of the travel distance of the vertical sample stage, and it was successfully evaluated within approximately $\pm 0.1 \mathrm{~mm}$ accuracy by image analysis for selected markers with higher contrast and higher circularity. Furthermore, concrete deformation under pullout loading applied to the embedded rebar was evaluated by the same procedure and the compressive deformation of the concrete part was successfully observed by analyzing the displacement of the Gd marker images. The measurement accuracy of the Gd marker displacement can be improved by choosing a spherical shaped Gd marker, by increasing the contrast of the Gd marker and also by increasing statistics accuracy. This method is expected to be utilized for clarifying the bond mechanism between concrete and rebar by observing concrete deformation around rebar combined with the bond stress distribution measured by the neutron diffraction technique.

\section{Acknowledgement}

These measurements at J-PARC MLF were performed under a user program (Proposal No. 2016B0155). This work was supported by JSPS KAKENHI Grant Number 17K05125. We would like to acknowledge Mr. K. Satake of The University of Tokyo for his experimental assistance.

\section{References}

[1] H. Suzuki, K. Kusunoki, Y. Hatanaka, T. Mukai, A. Tasai, M. Kanematsu, K. Kabayama and S. Harjo, Measuring strain and stress distributions along rebar embedded in concrete using time-of-flight neutron diffraction, Meas. Sci. Technol. $25 \quad$ (2014) 025602. https://doi.org/10.1088/0957-0233/25/2/025602

[2] H. Suzuki, K. Kusunoki, M. Kanematsu, A. Tasai, Y. Hatanaka, N. Tsuchiya, S.C. Bae, S. Shiroishi, S. Sakurai, T. Kawasaki and S. Harjo, Application of neutron stress measurement to reinforced concrete structure, JPS Conf. Proc. $8 \quad$ (2015) 031006. https://doi.org/10.7566/JPSCP.8.031006

[3] H. Suzuki, K. Kusunoki, M. Kanematsu, T. Mukai and S. Harjo, Structural engineering studies on reinforced concrete structure using neutron diffraction, Mater. Res. Proc. 2 (2016) 25-30.

[4] K. Watanabe, H. Higashi, T. Miki and J. Niwa, Real Time Image Analyzing System for loading tests of structural concrete, Doboku Gakkai Ronbunshuu E 66 (2010) 94-106 [in Japanese]. https://doi.org/10.2208/jsceje.66.94

[5] K. Yu, J. Yu, Z. Lu and Q. Chen, Fracture properties of high-strength/high-performance concrete (HSC/HPC) exposed to high temperature, Mater. Struct. 49 (2016) 4517-4532. https://doi.org/10.1617/s11527-016-0804-x

[6] T. Shinohara, T. Kai, K. Oikawa, M. Segawa, M. Harada, T. Nakatani, M. Ooi, K. Aizawa, H. Sato, T. Kamiyama, H. Yokota, T. Sera, K. Mochiki and Y. Kiyanagi, Final design of the Energy-Resolved Neutron Imaging System "RADEN" at J-PARC, J. Phys. Conf. Ser. 746 (2016) 012007. https://doi.org/10.1088/1742-6596/746/1/012007

[7] C.A. Schneider, W.S. Rasband and K.W. Eliceiri, NIH Image to ImageJ: 25 years of image analysis, Nat. Methods 9 (2012) 671-675. https://doi.org/10.1038/nmeth.2089 